\title{
Diffusion with random distribution of static traps
}

\author{
G. T. Barkema ${ }^{1}$, Parthapratim Biswas ${ }^{2}$, Henk van Beijeren ${ }^{1}$ \\ Theoretical Physics ${ }^{1}$, Utrecht University, Leuvenlaan 4, 3584 CE Utrecht, the Netherlands \\ Debye Institute ${ }^{2}$, Utrecht University, Princetonplein 5, 3508 TA Utrecht, the Netherlands
}

(November 2, 2018)

The random walk problem is studied in two and three dimensions in the presence of a random distribution of static traps. An efficient Monte Carlo method, based on a mapping onto a polymer model, is used to measure the survival probability $P(c, t)$ as a function of the trap concentration $c$ and the time $t$. Theoretical arguments are presented, based on earlier work of Donsker and Varadhan and of Rosenstock, why in two dimensions one expects a data collapse if $-\ln [P(c, t)] / \ln (t)$ is plotted as a function of $\sqrt{\lambda t} / \ln (t)$ (with $\lambda=-\ln (1-c)$ ), whereas in three dimensions one expects a data collapse if $-t^{-1 / 3} \ln [P(c, t)]$ is plotted as a function of $t^{2 / 3} \lambda$. These arguments are supported by the Monte Carlo results. Both data collapses show a clear crossover from the early-time Rosenstock behavior to Donsker-Varadhan behavior at long times.

The problem of trapping in a random medium is fundamental for understanding many important physical processes in disordered systems. Practical applications include some dynamical processes in disordered media, kinetics of reactions, electron-hole recombinations in random and amorphous solids, exciton trapping and annihilation, etc. 11. In the lattice version of this problem, traps are randomly distributed on a lattice of dimension d. Usually, all correlations between traps are ignored, largely motivated by the fact that in most relevant physical situations the concentration of traps is small. Initially at time $t=0$, a large population of random walkers is uniformly distributed over the lattice. Each walker hops from one lattice site to a randomly chosen nearestneighbor site, at a rate of one hop per time unit. When a walker meets a trap, it dies. The time dependence of the survival probability of the walkers in the presence of traps is an interesting problem, which has been analyzed both mathematically and numerically in the literature [1] 15]. The problem is related to the properties of the density of states of the electrons on a lattice with randomly distributed impurities, which has been discussed in Refs. 11, 12, 16, 17.

Theoretical treatments of the trapping problem usually start by considering the configuration averaged survival probability $P(c, t)$, where $c$ is the concentration of static traps. If we assign a label $i=1 \ldots N$ to all different random walks of $t$ steps and introduce $n(i, t)$ as the number of different sites visited by the walk labeled $i$ we can write the survival probability as

$$
P(c, t)=\left\langle(1-c)^{n(i, t)}\right\rangle,
$$

since the probability that a site is not a trap is equal to $(1-c)$. For short times $P(c, t)$ is well approximated by the
Rosenstock (RS) [6] expression $(1-c)^{\langle n(i, t)\rangle}$. This leads to exponential dependence on $t$ for $d \geq 3$ and exponential dependence on $\sqrt{t}$ in $d=1$. The short-time limit in two dimensions will be discussed further on in this paper.

The behavior of the survival probability at long times has been treated rigorously by Donsker and Varadhan 四; the limit $t \rightarrow \infty$ is known in the literature as DonskerVaradhan (DV) limit. In the DV limit, the survival probability $P(c, t)$ in $d$ dimensions does not decay exponentially, as simple intuition would suggest, but in a rather more complicated way:

$$
\ln [P(c, t)] \approx-A \lambda^{\frac{2}{d+2}} t^{\frac{d}{d+2}},
$$

where $A$ is a constant depending on dimension as well as on the characteristics of the lattice and the random walk process, and $\lambda=-\ln (1-c)$, with $c$ the concentration of static traps. An interpretation of this apparently unusual behavior was given by Grassberger and Procaccia [5]: they assign it to the existence of very rare large trap-free regions where walkers can survive for a long time. With increasing time ever larger trap free regions become dominant; the probability of finding such regions decreases exponentially with their $d$-dimensional volume, but the decay rate of particles moving within such a region is inversely proportional to the square of its diameter. The optimal choice of this diameter gives rise to the stretched exponential behavior of Eq. (2).

One important problem with the DV result is that it does not say anything about how large $t$ should be, in order to observe this behavior, nor what should be the corresponding value of the survival probability. The issue of the onset of the DV behavior has been studied numerically by a variety of algorithms, resulting in estimates which vary widely, apparently depending on the kind of algorithms used in the calculation. Earlier results of Klafter et al. [7], based on their numerical simulations, suggest that $P(c, t)$ should be less than $10^{-21}$ for $d=2$ to observe this behavior, whereas Fixman [8] obtained a value of $P(c, t)<10^{-67}$ in $d=3$. Among others, Havlin et al. [9] used an exact enumeration approach and obtained $P(c, t) \lesssim 10^{-13}$ for both two and three dimensions. The scanning method of Meirovitch [10] supported the claims of Havlin et al. None of the above studies have shown conclusively the crossover from the early-time RS behavior to the asymptotic DV behavior, except perhaps at very high trap densities. The most recent progress comes from Gallos et al. [14, who have studied the problem numerically in $d=2$, using the idea of self-interacting random walks coupled with a 
"slithering snake" algorithm. They plotted the quantity $-t^{-0.1} \ln [P(c, t)]$ as a function of $t^{0.8} \lambda$ and obtained a fairly satisfactory collapse, within their numerical precision; the theoretical basis for this kind of data collapse is however unclear.

Our algorithm for the symmetric random walk on a simple cubic or quadratic lattice, the case studied mostly before, is also based on the identification of each random walk of $t$ steps with a polymer configuration of length $t$. A direct Monte Carlo procedure to estimate the survival probability would then be to generate a large number of random walks, and average the quantity $(1-c)^{n(i, t)}$. This procedure would however give poor statistics with increasing $t$, since the result would be dominated by the one or two random walks that are the most compact. More accurate Monte Carlo results can be obtained by biasing the random walks generated towards more compact ones. Here, the identification of random walks with polymers comes into play. To each polymer configuration $i$ we attribute an energy, given by the Hamiltonian

$$
H(i, t)=\lambda n(i, t) .
$$

If we then sample polymer configurations at infinite temperature, the average energy is equal to $\lambda n(i, t)$, which in turn equals $\ln [P(c, t)]$. Numerically more accurate results are obtained, however, by generating polymer configurations at some inverse temperature $\beta$. The likelihood for a configuration $i$ to be generated is then proportional to its Boltzmann factor $\exp (-\beta H(i, t))$ and as a consequence there is a bias towards compact configurations. Also from this ensemble, one can compute the survival probability using

$$
P(c, t)=\frac{\left\langle\exp (+\beta H(i, t))(1-c)^{n(i, t)}\right\rangle_{\beta}}{\langle\exp (+\beta H(i, t))\rangle_{\beta}}
$$

where the averaging is performed over the ensemble generated at inverse temperature $\beta$. Note that at infinite temperature, i.e. $\beta=0$, there is no bias and we retrieve Eq.(11). Interestingly, at inverse temperature $\beta=1$ the numerator equals unity and the inverse of the survival probability equals the average of the inverse Boltzmann weights. It turns out that the most accurate results are obtained at some inverse temperature between 0 and 1 ; all our results are obtained at $\beta=0.75$.

We generate a Markov chain of polymer configurations at inverse temperature $\beta$ by proposing randomly chosen single-monomer moves, and accepting each proposed move with the Metropolis acceptance probability

$$
P_{a c c}=\min [1, \exp (-\beta \Delta E)],
$$

where $\Delta E$ is the change in energy, defined by the Hamiltonian Eq. (3). The types of single-monomer moves that we propose are depicted in Fig. 1. In a naive implementation, the computational effort per move would scale linearly with polymer length because of the necessity to walk along the polymer to compute the change in energy.
However, if one stores in each lattice site the number of times the polymer visits that site, then the energy difference can be computed easily: the energy increases by $\lambda$ if the monomer moves to a site which is not visited before, decreases by $\lambda$ if the monomer leaves a site that it visits only once, and stays unaltered if either both or neither of these two situations hold. This administration is also easily updated: the new site is visited one more time, while the old site is visited one time less.

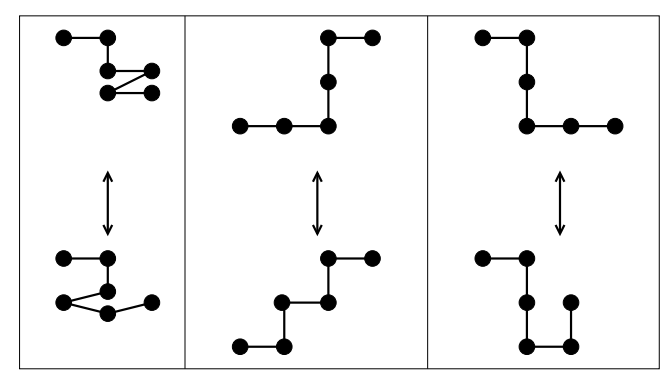

FIG. 1. Monte Carlo moves used in the simulation: exchange of a pair of consecutive steps (left and middle), or replacement of the first or last step (right).

We now discuss numerical results obtained using our algorithm outlined above. First, we concentrate on the case of two dimensions. In Fig. 2, we have plotted the logarithm of the survival probability $P(c, t)$ for concentrations $c=0.01,0.02, \ldots, 0.09 ; 0.1,0.2, \ldots, 0.9$, and times (polymer lengths) up to $t=825$. In these simulations, we start with a random walk, which is then simulated over $10^{9}$ Monte Carlo steps per monomer, at an inverse temperature of $\beta=0.75$. Data obtained during the first $50000 t$ attempted Monte Carlo steps per monomer have been discarded (thermalization).

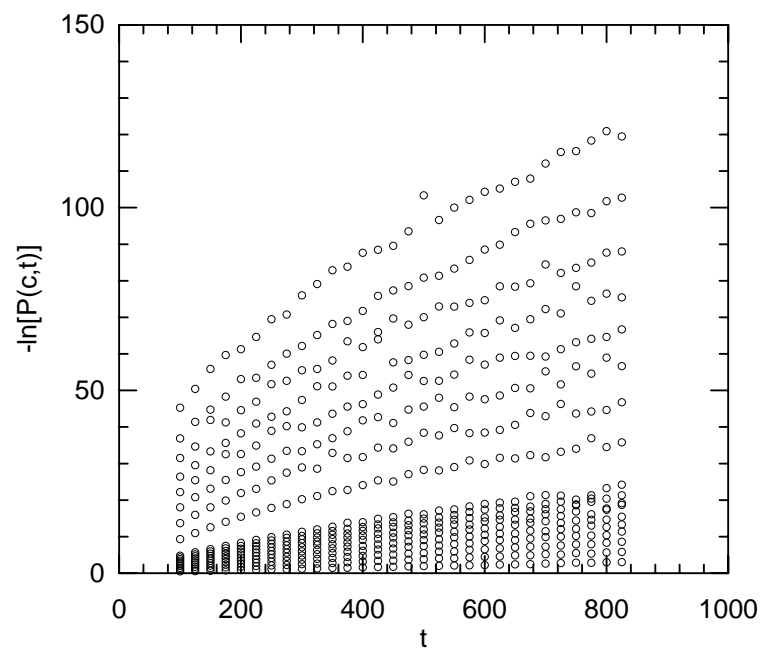

FIG. 2. Monte Carlo results for the survival probability as a function of time, for several trap concentrations on a two-dimensional lattice: $-\ln [P(c, t)]$ is plotted as a function of time $t$. 
We now obtain a theoretically justified data collapse based on the identification of a common scaling variable for the DV and RS regimes. At short times and small concentrations, we expect that the polymer configuration closely resembles a random walk. In the case of two dimensions, this means that the number of different sites visited scales as $t / \ln (t)[18]$. Consequently, we deduce that the RS behavior in two dimensions is properly described by

$$
-\ln [P(c, t)] \sim \lambda t / \ln (t) .
$$

At long times and large concentrations we expect to observe the DV behavior:

$$
-\ln [P(c, t)] \sim(\lambda t)^{1 / 2} .
$$

To obtain the proper scaling variable, we equate the right terms in equations (6) and (6), and obtain $\sqrt{\lambda t} / \ln (t)=1$; we can use this as a scaling variable, and rewrite equations (6) and (7) as:

$$
\begin{aligned}
& \frac{-\ln [P(c, t)]}{\ln (t)}=\left[\frac{\sqrt{\lambda t}}{\ln (t)}\right]^{2} ; \\
& \frac{-\ln [P(c, t)]}{\ln (t)}=\frac{\sqrt{\lambda t}}{\ln (t)} .
\end{aligned}
$$

Thus we expect that if $-\ln [P(c, t)] / \ln (t)$ is plotted as a function of $\sqrt{\lambda t} / \ln (t)$, the data for all values of trap concentration $c$ and time $t$ collapse onto a single curve, with an effective exponent that crosses over from 2 to 1 .

In Fig. 3 we have performed this plot, using the same data as in Fig. 2. Clearly, the data collapse is convincing over the whole range of parameters used in our simulation. Also, one can clearly identify the RS regime with a slope of 2 and the DV regime with a slope of 1 , both indicated with solid lines. The numerical estimate of the point where these lines cross is $-\ln [P(c, t)] / \ln (t)=3.5$ and $\sqrt{\lambda t} / \ln (t)=1.13$. The survival probability where the DV regime starts is then given by $-\ln [P(c, t)]=$ $3.1 \sqrt{\lambda t}$, for a suitable choice of $c$ and $t$. The fact that the survival probability at the start of the DV regime is not a constant explains the wide range of reported values for this quantity. The apparent dependence of the results on the simulation methods can be understood because certain simulation methods are especially suited for high $c$-values whereas other methods require fairly low values of $c$. From our results one may conclude that the DV regime can already start at a significant survival probability, depending on $c$; it should therefore be observable experimentally.

To illustrate this in detail we plotted in Fig. 1 the cross-over values of both time and survival probability as functions of the trap concentration.

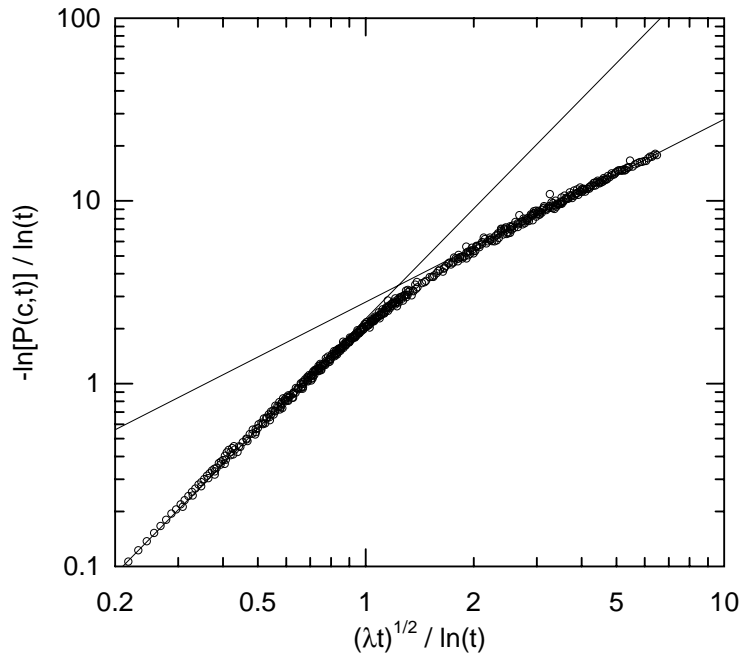

FIG. 3. Collapse of the two-dimensional data: $-\ln [P(c, t)] / \ln (t)$ is plotted as a function of $\sqrt{\lambda t} / \ln (t)$ in a double-logarithmic plot. The solid lines are fits to the data, with slopes 2 and 1 . They cross at the point $(1.13,3.5)$.

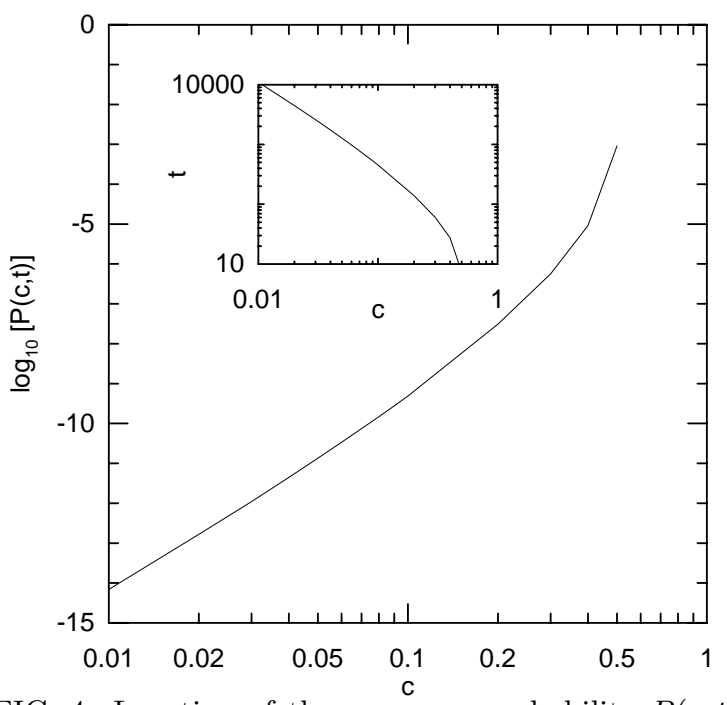

FIG. 4. Location of the crossover probability $P(c, t)$ as a function of trap concentration $c$, in two dimensions. The inset shows the crossover time $t$ as a function of $c$.

In dimensions different from $d=2$ it is equally possible to define a common scaling variable for the RS and DV regimes, that ought to give rise to a data collapse. For $d<2$ one should consider $\ln [P(c, t)]$ as a function of $(\lambda t)^{d / 2}$ and for $d>2$ collapse should occur for $-t^{(2 / d-1)} \ln [P(c, t)]$ as a function of $t^{2 / d} \lambda$. From these expressions one first of all sees very clearly that $d=2$ acts as a cross-over value for the dimensionality, as suggested already by the logarithmic terms in the $2 d$ scaling variables.

In particular, in three dimensions, where the number of different sites visited increases linearly with time $t$, the RS and DV regimes can be written as:

$$
-t^{-1 / 3} \ln [P(c, t)]=t^{2 / 3} \lambda ;
$$




$$
-t^{-1 / 3} \ln [P(c, t)]=\left[t^{2 / 3} \lambda\right]^{2 / 5} .
$$

To show the resulting data collapse in three dimensions, we plotted in Fig. $0 t^{-1 / 3} \ln [P(c, t)]$ as a function of $t^{2 / 3} \lambda$. All data have been obtained again with the polymer algorithm described above. As in two dimensions, they can be explained by a crossover from the RS regime, where the curve has a slope of 1 , to the DV regime, where the curve has a slope of $2 / 5$.

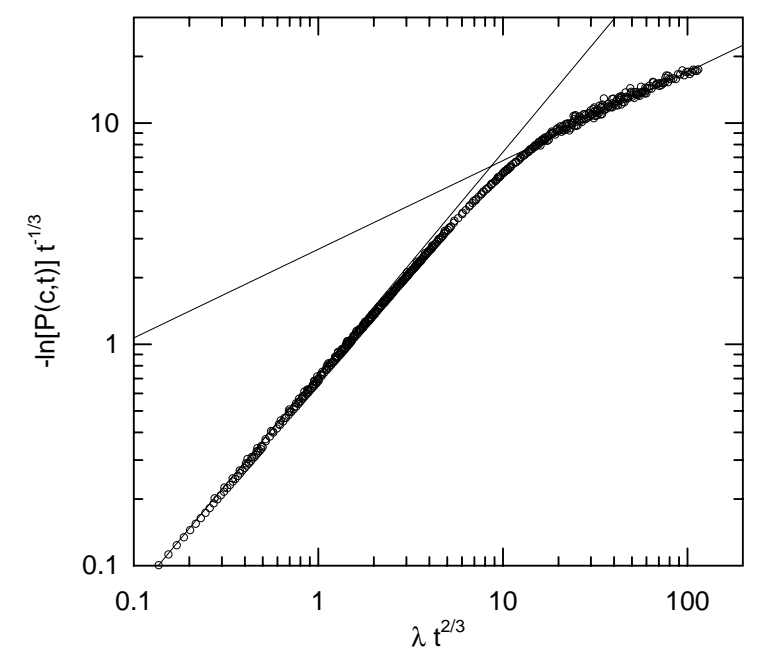

FIG. 5. Collapse of the three-dimensional data: $-t^{-1 / 3} \ln [P(c, t)]$ is plotted as a function of $t^{2 / 3} \lambda$ in a double-logarithmic plot. The solid lines are fits to the data, with slopes 1 and $2 / 5$. They cross at the point $(8.5,6.3)$.

In Fig. 6 we also plotted the cross-over time and survival probability as functions of $c$ for three dimensions. As one might expect, the concentration dependence is even much stronger than in two dimensions.

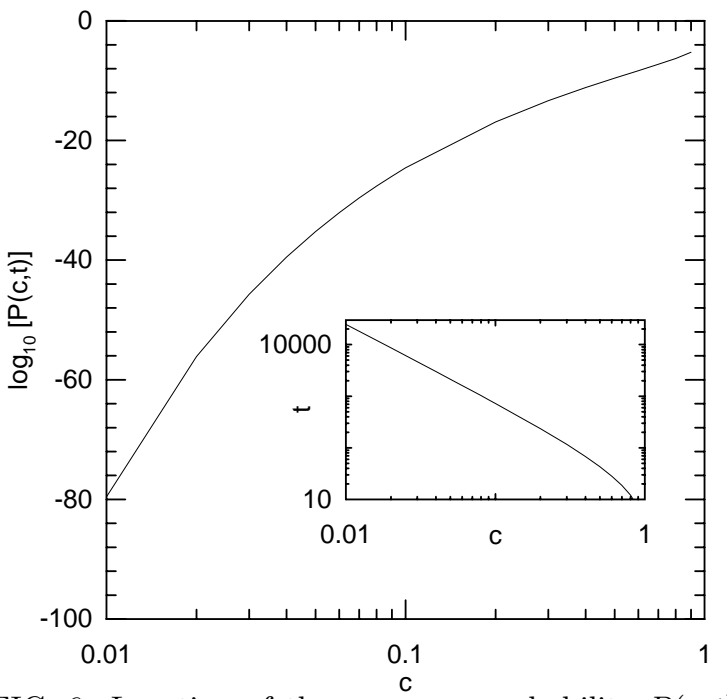

FIG. 6. Location of the crossover probability $P(c, t)$ as a function of trap concentration $c$, in three dimensions. The inset shows the crossover time $t$ as a function of $c$.
In summary, we have outlined an efficient algorithm to investigate diffusion with random traps in an arbitrary concentration range. The simulation results for all trap concentrations and times could be explained by a crossover from the Rosenstock behavior at short times to the Donsker-Varadhan behavior at long times. We have identified the location of the crossover point, which in contrast to earlier belief, does not simply yield a specific value for the survival probability.

[1] S. Havlin, D. Ben-Avraham, Adv. Phys. 36, 696 (1987) and references therein.

[2] J. R. Beeler, Phys. Rev. A 134, 1396 (1964).

[3] J. Haus and K. W. Kehr, Phys. Rep. 150, 263 (1987).

[4] M. Donsker and S. R. S. Varadhan, Commun. Pure Appl. Math. 28, 525 (1975); ibid 32, 721 (1979).

[5] P. Grassberger and I. Procaccia, J. Chem. Phys. 77, 6281 (1982).

[6] H. B. Rosenstock, J. Math. Phys. 11, 487 (1970).

[7] J. Klafter, G. Zumofen and A. Blumen, J. Phys.(Paris) 45, L49 (1984).

[8] M. Fixman, Phys. Rev. Lett. 52, 791 (1984).

[9] S. Havlin et. al, Phys. Rev. Lett. 53, 407 (1984).

[10] H. Meirovitch, Phys. Rev. A 32, 3699 (1985).

[11] Th. M. Nieuwenhuizen. Phys. Rev. Lett. 62, 357 (1989).

[12] T. C. Lubensky, Phys. Rev. A 30, 2657 (1984); A. B. Harris and T. C. Lubensky, Phys. Rev. B 23, 2640 (1981).

[13] J. K. Anlauf, Phys. Rev. Lett. 52, 1845 (1984).

[14] L. K. Gallos, Panos Argyrakis and K. W. Kehr, Phys. Rev. E 63, 21104 (2001).

[15] F. den Hollander and G. H. Weiss, in Contemporary Problems in Statistical Physics, edited by G. H. Weiss (SIAM, Philadelphia, 1994).

[16] I. M. Lifshitz, Usp. Fiz. Nauk. 83, 617 (1964); Sov. Phys. Usp. 7, 549 (1965).

[17] R. Friedburg and J. M. Luttinger, Phys. Rev. B 12, 4460 (1976).

[18] E. W. Montroll and G. H. Weiss, J. Math. Phys. 6, 167 (1965); J. W. Haus and K. W. Kehr, Phys. Rep. 150, 263 (1987).

[19] N. Metropolis, A. W. Rosenbluth, M. N. Rosenbluth, A. H. Teller, and E. Teller, J. Chem. Phys. 21, 1087 (1953). 